(a)

CrossMark click for updates

Cite this: Polym. Chem., 2016, 7, 1682

DOI: $10.1039 /$ c6py90024k

www.rsc.org/polymers

\section{Correction: Chemistry of aromatic polythioesters and polydithioesters}

\author{
Daisuke Abe, Yuichiro Fukuda and Yuji Sasanuma*
}

Correction for 'Chemistry of aromatic polythioesters and polydithioesters' by Daisuke Abe, et al., Polym. Chem., 2015, 6, 3131-3142.

The authors regret the wrong arrangement of the trans fractions derived from $\mathrm{MO}$ calculations for the $\mathrm{S}_{-} \mathrm{CH}_{2}$ bond in Table 1 of the original paper. The corrected version of Table 1 is as shown below.

Table 1 Vicinal coupling constants and trans fractions $\left(p_{\mathrm{t}}{ }^{\prime} \mathrm{s}\right)$ of $3 \mathrm{DBS}_{2}$ and $3 \mathrm{DBS}_{4}$

\begin{tabular}{|c|c|c|c|c|c|c|c|}
\hline \multirow[b]{2}{*}{ Medium } & \multirow[b]{2}{*}{ Temp. $\left({ }^{\circ} \mathrm{C}\right)$} & \multirow[b]{2}{*}{${ }^{3} J_{\mathrm{HH}}{ }^{a}$} & \multirow[b]{2}{*}{${ }^{3} J_{\mathrm{HH}}^{\prime}{ }^{a}$} & \multirow[b]{2}{*}{${ }^{3} J_{\mathrm{CH}}{ }^{a}$} & \multicolumn{3}{|l|}{$p_{\mathrm{t}}$} \\
\hline & & & & & Set $\mathrm{A}^{b}$ & Set $B^{c}$ & $\mathrm{~S}-\mathrm{CH}_{2}{ }^{d}$ \\
\hline \multicolumn{8}{|l|}{$3 \mathrm{DBS}_{2}(\mathrm{NMR}$ expt$)$} \\
\hline \multirow{3}{*}{ Cyclohexane- $\mathrm{d}_{12}$} & 15 & 6.61 & 7.50 & 4.55 & 0.42 & 0.44 & 0.14 \\
\hline & 25 & 6.62 & 7.47 & 4.53 & 0.42 & 0.44 & 0.15 \\
\hline & 55 & 6.72 & 7.46 & 4.46 & 0.41 & 0.43 & 0.16 \\
\hline Benzene- $\mathrm{d}_{6}$ & 25 & 6.47 & 7.84 & 4.46 & 0.45 & 0.47 & 0.16 \\
\hline \multicolumn{8}{|l|}{$3 \mathrm{DBS}_{2}(\mathrm{MO}$ calc.) } \\
\hline \multirow[t]{3}{*}{ Gas } & 15 & & & & 0.44 & & 0.11 \\
\hline & 25 & & & & 0.44 & & 0.12 \\
\hline & 35 & & & & 0.44 & & 0.12 \\
\hline \multirow{5}{*}{ Cyclohexane- $\mathrm{d}_{12}$} & 15 & 6.48 & 7.89 & 5.40 & 0.47 & 0.47 & 0.20 \\
\hline & 25 & 6.50 & 7.89 & 5.37 & 0.46 & 0.47 & 0.20 \\
\hline & 35 & 6.50 & 7.88 & 5.34 & 0.46 & 0.47 & 0.21 \\
\hline & 45 & 6.54 & 7.84 & 5.30 & 0.46 & 0.46 & 0.21 \\
\hline & 55 & 6.54 & 7.83 & 5.27 & 0.46 & 0.46 & 0.22 \\
\hline Benzene- $\mathrm{d}_{6}$ & 25 & 6.43 & 8.14 & 5.25 & 0.48 & 0.49 & 0.22 \\
\hline \multicolumn{8}{|l|}{$3 \mathrm{DBS}_{4}(\mathrm{MO}$ calc. $)$} \\
\hline \multirow[t]{5}{*}{ Gas } & 15 & & & & 0.38 & & 0.19 \\
\hline & 25 & & & & 0.38 & & 0.19 \\
\hline & 35 & & & & 0.38 & & 0.20 \\
\hline & 45 & & & & 0.38 & & 0.20 \\
\hline & 55 & & & & 0.38 & & 0.21 \\
\hline Benzene & 25 & & & & 0.41 & & 0.22 \\
\hline
\end{tabular}

The Royal Society of Chemistry apologises for these errors and any consequent inconvenience to authors and readers.

Department of Applied Chemistry and Biotechnology, Graduate School and Faculty of Engineering, Chiba University, 1-33 Yayoi-cho, Inage-ku, Chiba 263-8522, Japan. E-mail: sasanuma@faculty.chiba-u.jp; Fax: +81-43-290-3394; Tel: +81-43-290-3394 